\title{
SISTEMA INTELIGENTE DE TRIAGEM PARA SEPARAÇÃO DE GARRAFAS PET COM REDES NEURAIS ARTIFICIAS ${ }^{1}$
}

\author{
ALICLECIO OLÍMPIO DA SILVA², IVAN NUNES DA SILVA ${ }^{3}$
}

${ }^{1}$ Título do Trabalho de Redes Neurais Artificiais

${ }^{2}$ Autor,Aluno Especial do Curso de Mestrado em Sistemas Inteligentes

${ }^{3}$ Professor Orientador, Bacharel em Ciência da Computação e Engenharia Elétrica, Mestre e Doutor em Estudos de Redes

Neurais Artificiais

Laboratório de Automação Inteligente de Processos e Sistemas, USP-EESC Av.

Trabalhador São-carlense, 400-CEP 13566-590, São Carlos-SP

E-mails: $\{$ aliander476@gmail.com, insilva\}@sel.eesc.usp.br\}

\begin{abstract}
This work presents the construction of an intelligent system based on neural networks for the use of PET bottle sorting and sorting through the standard colors of PET bottles in a recycling center, this system will automate in an intelegent way what is now done with the help of mats and with the help of people to separate the bottles by the specific colors before they are crushed for the confection of new materials from these new compositions.
\end{abstract}

Keywords- Artificial neural networks, intelligent system, recycling, PET bottle.

Resumo - Este trabalho apresenta a construção de um sistema inteligente a base de redes neurais para uso de triagem e separação de garrafas PET através das cores padrões das garrafas PET, em uma central de reciclagem, esse sistema irá automatizar de forma inteleginte o que hoje é feito com a ajuda de esteiras e com auxílio de pessoas para separa as garrafas pelas cores especifica antes de serem trituradas para a confexão de novos matérias a partir dessas novas composições.

Palavras-chave-Redes neurais artificiais, sistema inteligente, reciclagem, garrafa PET.

\section{Introdução}

A Reciclagem de garrafas PET no Brasil é uma das mais desenvolvidas no mundo. Conta com alto índice de reciclagem e uma enorme gama de aplicações para o material reciclado, criando uma demanda constante e garantida. Atualmente, a indústria recicladora está estabelecida por todo território nacional, o sistema constituem de catadores individuais, cooperativas de reciclagem e grandes centros de triagem desses materiais, que posteriormente são utilizados em diversos seguimento, desde confexão de roupas outros tios de embalagens, tintas, peças para área automobilista e outras infinidades de materiais (fonte, ABIPET), atualmente a maior parte do processo é feito de maneira manual um processo de
Formiguinha onde requer muitas passos envolvidas, o Processo de triagem de separação de garrafas PET se dar através das cores basicamente existem 3 cores padrões de garrafas PET mais usados no brasil, transparente ou incolor, verde e azul (fonte, ABIPET), o processo de triagem passa por várias etapas até a separação final ou processo de triagem é feito por esteiras e várias pessoas separando por cores, a ideia e criar um centro de triagem a base de redes neurais artificiais que faça de forma automático esse processo de separação e outras etapas do processo também, para que as PET separadas por cores para cerem trituradas e colocadas em sacos embalagens para serem destinadas a diversas fabricações de novos produtos, assim ganhando um processo mais rápido eficiente e menos cansativo. 


\section{Redes Neurais Artificias}

Redes neurais artificiais é um conceito da computação que visa trabalhar o processamento dedados de maneira semelhante ao cérebro humano. Nas redes neurais artificiais, a ideia é realizar o processamento das informações tendo como princípio a organização de neurônios do cérebro. Assim, uma rede neural pode ser interpretada como um esquema de processamento capaz de armazenar conhecimento baseado em aprendizagem (experiência) e disponibilizar este conhecimento para a aplicação em questão. As redes neurais artificiais (RNAs) já estão definitivamente consolidadas como uma alternativa à computação programada tradicional na solução de problemas complexos que demandam a análise de grandes volumes de dados. Foram aplicadas em quase todos os ramos do conhecimento humano.

\subsection{Histórico}

As redes neurais artificiais são relativamente novas, os primeiros modelos de redes neurais que se tem relatos foram criadas em 1943, através da primeira publicação relacionada a neurocomputação

Por meio do artigo elaborado por McCulloch \& Pitts (1943). Nesse trabalho apresentou-se um modelamento baseado no neurônio biológico, criando assim o neurônio artificial.

Em 1949 o psicólogo canadense Donald Olding Hebb, criou o primeiro método de treinamento de redes neurais artificiais que ficou conhecido como regra de aprendizado de Hebb. Este aprendizado baseou-se em hipóteses e observações de caráter neurofisiológico [HEBB, 1949].

Na década de 1950 os estudos em redes neurais continuaram a serem avançando, criando-se uma nova série de topologias e algoritmos de aprendizado, nessa década com vários trabalhos se destaca o trabalho do psicólogo americano Frank Rosenblatt, que entre 1957 e 1958, desenvolveu o primeiro neurocomputador, chamado Mark I perceptron, [ROSENBLATT, 1958], nasce ai umas das redes neurais mais importantes e utilizadas até os dias de hoje.

Já na década de 1960, após criar-se o perceptron surgiu vários trabalhos e interesse em desenvolver outros tipos de redes neurais, pelo motivo que peceptron ter grande capacidade de reconhecer padrões simples, foi então que o engenheiro e professor americano Bernad Windrow e seu aluno de doutorado Marcian Hoof criaram em 1960 uma rede chamada de Adaline, (ADApiteve LINear Element, e depois o Madaline (Adaline mútipla) [WINDROW \& HOFF 1960] e criou-se também aprendizado chamdo de regra delta, o algoritimo LMS (Least mean square). Em 1969 com o sucesso do Perceptron e o Adaline sugiram mais pesquisadores afim de estudar outros modelos de redes, e também com alguns problemas e críticas em relação as redes neurais, com a publicação do famoso livro Perceptrons, pelo cientista americano Marvin Minsky e pelo matemático sul-africano Seymour Papert desmostram as limitações das redes neurais criadas até então, constituídas apenas de uma camada, que não conseguiam classificar amostras com classes não linearmente separáveis. Daí surgiu o problema da lógica XOR (ou-exclusivo).

Já na década de 1970 , os estudos de rede neurais deu uma pequena esfriada com pouquíssimas pesquisas foram desenvolvida, a única de mais destaque foi a derivação de algoritmo de predição utilizado gradiente reverso, [WERBOS, 1974], na década de 1980 os estudos e pesquisas com redes neurais voltaram a ter destaques e surgiram novas apresentações e novos projetos, no ano de 1980 o neurocientista Sphen Grossberg fez a implementação da red ART(adaptive resonance tbeory), no ano de 1982 o cientista finlandês Teuvo Kohonen criaou as formulações do algoritimo de mapas auto-organizáveis de Kohonen, nesse mesmo ano físico e neurologista americano John Joseph Hopfild criou as redes recorrentes, baseadas em funções de energia. No final dos anos 1980 a publicação do livro de [Rumelhart et allii, 1986] em que os autores criarão redes com mais de uma camada, solucionando o caso da XOR, onde se permite ajustar os pesos para diminui o máximo os erros, a partir daí os estudos de redes neurais voltaram à tona e ainda se tem mais uma descoberta importante que foi o algoritmo backpropagation.

Na década de 1990, as redes neurais continuaram e crescimento aparecendo vários conceitos de redes $\mathrm{e}$ algoritmos alguns em destaque, para o algoritmo de Levenberg-Marquardt, permitindo incrementar e adicionar técnicas mais eficientes nos treinamentos das redes neurais (support vector machinesSVM),[HAFAN \& MENCHJ, 1994], e também a classificação de padrão regressão e linear [VAPNIK, 1998].

Na década de 2000 as redes neurais continuaram em evolução com implemento em circuitos integrados neurais com diversas configurações [BEIU ET, 2003], nos dias de hoje tem as rede convolucionais, deep learning, e redes muito mais poderosas re eficientes resolvendos cada vez mais para a resolução de vários problemas em vários seguimentos.

\subsection{Neurônio Biológico}

O processamento de informações no cérebro humano, operam de forma paralela tendo como objetivo a produção de ações apropriadas para cada uma de suas funcionalidades [I.N.SILVA, D.H.SPATTI, R.A. FLAUZINO, 2016]. O neurônio biológico possuem partituras que juntas cada executam algumas funções especificas o modelo de um neurônio biológico está representado na figura 1.0.

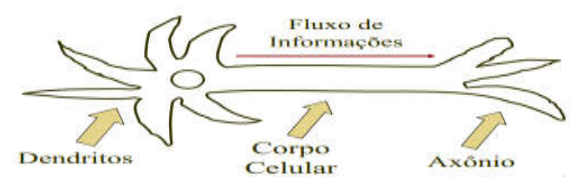

Figura 1.0 Neurônio biológico (I.N.Silva, D.H.Spatti, R.A. Flauzino

2.3 Neurônio Artificial 
O modelo estrutural básico das redes neurais artificiais foram inspirados a partir de sistemas nervosos biológicos e do próprio cérebro humano. Tais modelos foram criados a partir da análise da geração e propagação de impulsos elétricos pela membrana celular dos neurônios [HODGKIN \& HUXLEY, 1952]. A estrutura de um neurônio simples com as principais características de uma rede neural artificial, ainda mais usado é o modelo proposto por [MCCULLOCH \& PITTS,1943], essa representação e suas características são mostradas na figura 1.1.

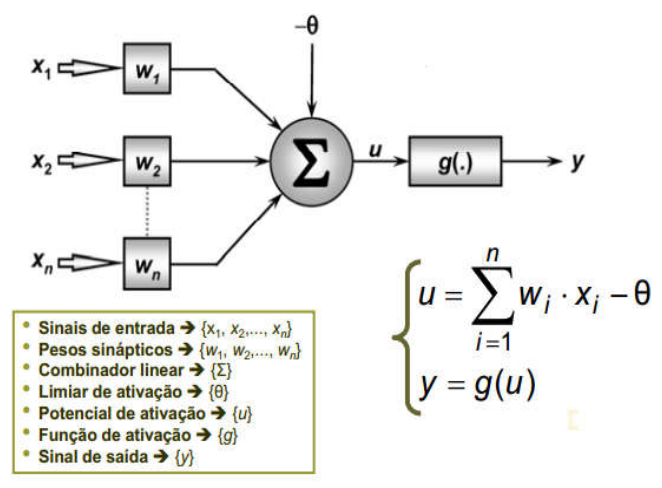

Figura 1.1 Neurônio artificial (I.N.Silva, D.H.Spatti, R.A. Flauzino)

\subsection{Arquiteturas de Redes Neurais Artificias}

As arquiteturas das redes neurais se dividem em três partes chamadas de camadas fundamentais para o funcionamento correto de uma rede, alguns possuem apenas duas dessas camadas, a figura 1.2 mostra um exemplo.

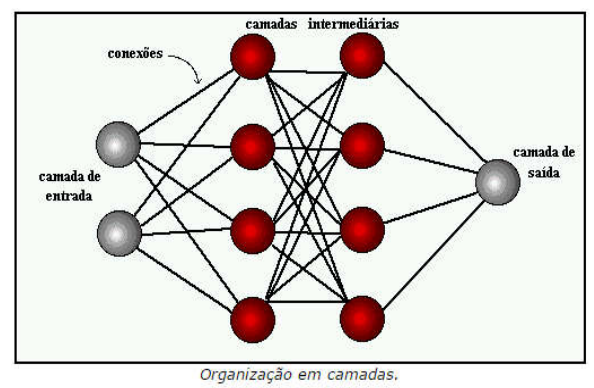

Figura 1.2 Exemplo representação das camadas de uma rede neural (ICM)

Camada de Entrada: onde os padrões são apresentados à rede.

Camadas Intermediárias ou Escondidas: onde é feita a maior parte do processamento, através das conexões ponderadas; podem ser consideradas como extratoras de características.

Camada de Saída: onde o resultado final é concluído e apresentado.

\subsubsection{Feedforword camada simples}

Esse modelo de arquitetura de rede possuem a camada de entrada e uma única camada, a de saída sem ter camadas intermediárias os neurônios são as próprias saídas a figura 1.3 mostra uma desta arquitetura.

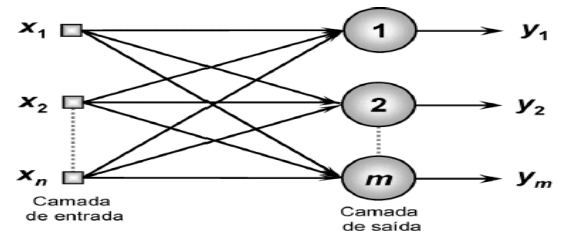

Figura 1.3 Feedforword simples(I.N.Silva, D.H.Spatti, R.A. Flauzino)

\subsubsection{Feedforword camadas múltiplas}

Já essa arquitetura são compostas de várias camadas incluindo as camadas ocultas diferentes da anterior, a figura 1.4 mostra uma desta arquitetura.

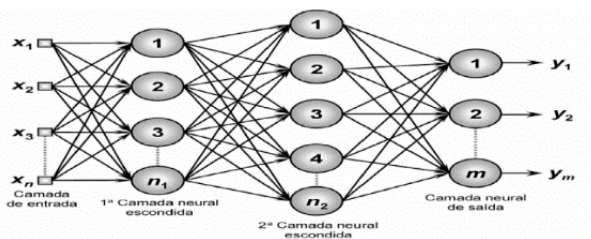

Figura 1.4 Feedforword camadas múltiplas(I.N.Silva, D.H.Spatti, R.A. Flauzino)

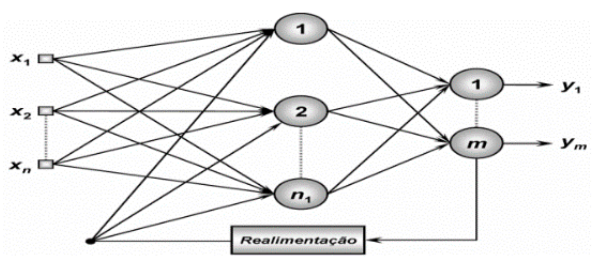

\subsubsection{Arquitetura recorrente}

Essa arquitetura é muito parecida com a anterior com a diferencia que tem uma realimentação da camada de saída comas camadas intermediárias existentes, como mostra a figura 1.5.

Figura 1.5 Rede recorrente (I.N.Silva, D.H.Spatti, R.A. Flauzino) 2.3.4 Arquitetura estruturada e reticulada

Essa arquitetura possui localização espacial de neurônio, essas redes podem serem usadas e reconhecimento de padrões, otimização de sistema etc. a figura 1.6 mostra esse tipo de arquitetura.

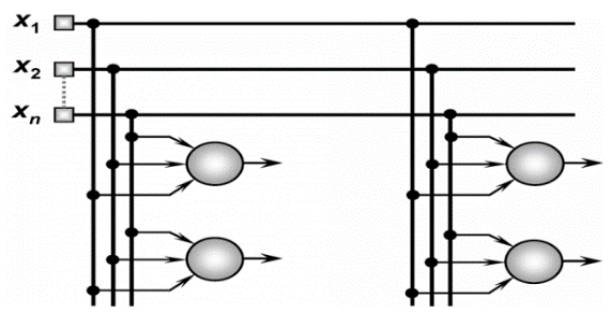


Figura 1.6 Rede Estruturada reticulada (I.N.Silva, D.H.Spatti, R.A. Flauzino)

\subsection{Treinamento e Aprendizado}

As redes neurais artificiais se destacam pelo grande poder de aprender a partir de apresentação de amostras padrões, o sucesso desse aprendizado dependem do treinamento que é processo de aplicação dos pesos ordenados necessários para a sintonização dos peso sinápticos e limiares de seus neurônios, [I.N.SILVA, D.H.SPATTIS, R.A.FLAUZINO, 2016].

\subsubsection{Treinamento supervisionado}

Neste tipo de treinamento, tem-se disponível e conhece os valores de saídas desejados, dessa forma há obrigação de disponibilizar tabelas de dados de entradas e saídas, assim treina a rede para o maior número de acertos, reduzindo o erro para valores aceitáveis, esse processo tem como se tivesse um 'professor' que confere os valores de saídas da rede para que ela tenha o maior número de acerto possível.

A primeira forma de treinamento supervisionado foi proposto por Donald Hebb, cujo desenvolvimento veio através de observações neurofisiológicas [HEBB, 1949].

\subsubsection{Treinamento não supervisionado}

O treinamento não supervisionado é o oposto do supervisionado, não se tem saídas desejadas, o algoritmo deve ser constituído a se auto organizar, através de subconjuntos (clausters), [I.N.SILVA, D.H.SPATTI, R.A. FLAUZINO, 2016].

\subsubsection{Treinamento com reforço}

Esse tipo de treinamento tem sido considerado como uma variação do treinamento supervisionado, onde o algoritmo é capaz de fazer uma defasagem de valor de resposta entre o valor produzido na saída da rede com valor desejado [SUTTONS \& BARTO, 1998]. No decorrer do processo treinamento, as probabilidades associadas às ações de ajustes são ainda modificadas visando melhorar o desempenho da rede [TSOUKALAS \& UHRI, 1997].

\subsubsection{Aprendizado por lote de padrão (off-line)}

O processo de aprendizado por lote de padrão ajustas os pesos e limiares após a apresentação de todo o conjunto de amostras

\subsubsection{Aprendizado usando padrão-por-padrão(on-line)}

O processo de aprendizado padrão-por-padrão ajustas os pesos e limiares após o final de cada apresentação de amostras.

3 Definição do problema
Quais os impactos e soluções que um sistema inteligente ajudará em uma triagem e separação de garrafas PET?.

\section{Solução do problema}

Como solução do problema, um sistema de triagem e separação de garrafas pet para reciclagem através das cores, existem basicamente 3 cores predominante e comercialmente fabricada de garrafas pet, Transparente, Verde e Azul, o é sistema constituído por um tanque d'água por gravidade onde são colocadas as garrafas pet transporta em sacos nesse primeiro estágio as embalagem são retiradas cortadas pelos estripadores e consequentemente são separadas das garrafas que seguirão para o segundo estágio que é esteira de entrada onde essas garrafas seguirão até a caçamba analisadoras onde estão os tubos de visualização que em sua entrada possui câmeras que analisarão as garrafas que entra no tubo e consequentemente enviarão os dados para a rede neural através do código RGB das cores primaria, essas informações são analisadas pela rede neural que separará as cores treinadas, acionando separando 4 classes, representando as quatros cores de garrafas, as mesma serão direcionada paras as suas respectivas esteiras através de quatros expulsadores e quatros esteiras para cada cores correspondente, transparente, verde, azul e outras. Passado esse processo cada esteira com as correspondentes cores das garrafas levarão até seus respequitivos moedores para moerem e assim o material final está pronto para serem ensacados e vendidos paras as empresas fabricam vários tipos de produtos à base de pet.

\section{Fluxograma do sistema}

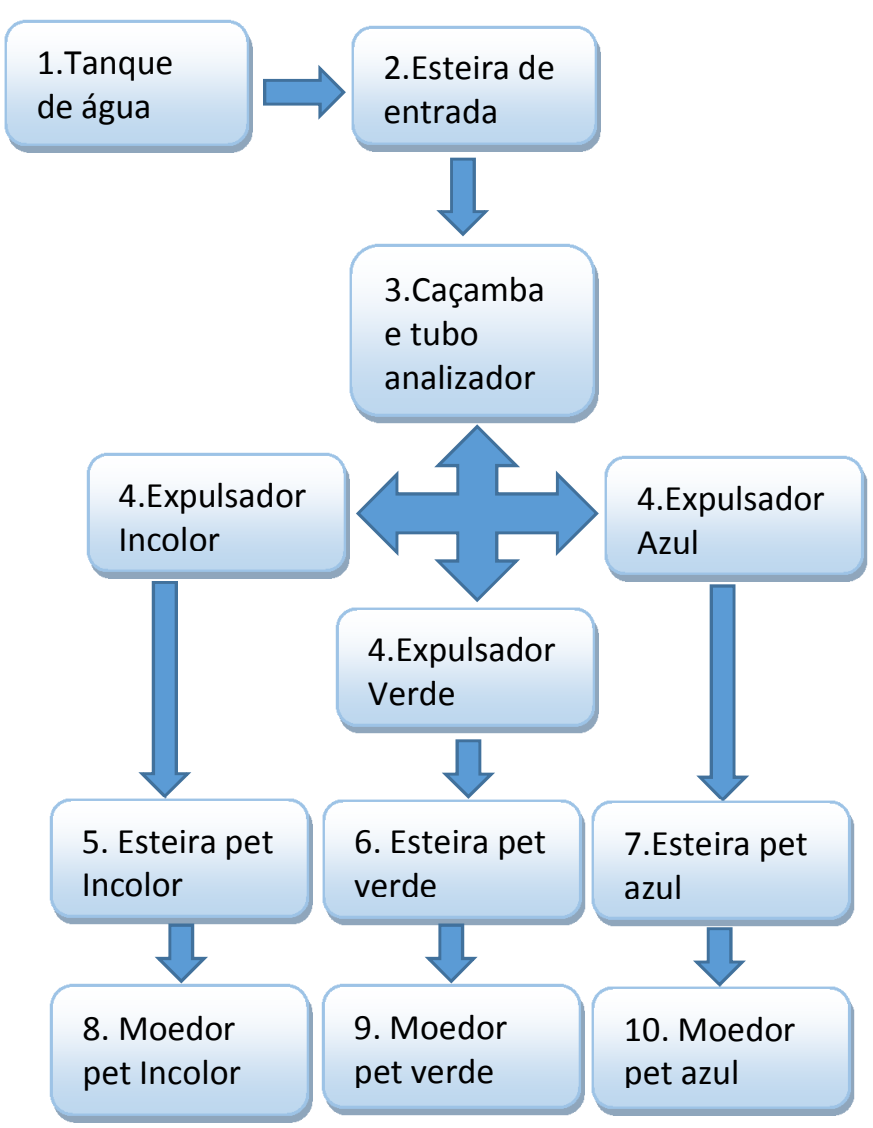


4.

\subsection{Dados da rede neural ultilizada}

Neste trabalho, a RNA empregada possui arquitetura com múltiplas camadas, denominada MLP (Multilayer Perceptron). A arquitetura MLP As redes neurais multicamadas são arquiteturas onde os neurônios são organizados em duas ou mais camadas de processamento, já que sempre vai existir pelo menos uma camada de entrada e uma camada de saída. Estas arquiteturas são as mais frequentemente encontradas na literatura referente a redes neurais artificiais.

As redes neurais com apenas duas camadas são constituídas de uma camada de entrada que se conecta a uma camada de neurônios de saída. Os neurônios da camada de entrada são neurônios especiais, cujo papel é exclusivamente distribuir cada uma das entradas da rede (sem modificá-las) a todos os neurônios da camada seguinte. A forma mais simples deste tipo De rede neural, consiste de um único neurônio na camada de saída, sendo conhecido como perceptron.

A configuração rede neural utilizada no trabalho possuem as seguintes caraterísticas.

1 Camada de Entrada: 4 camadas de atributos de cores dos 3 tipos de garrafas pet mais outro tipo.

2 Camada Escondida: Contempla 10 neurônios.

3 Camada de Saída: Contempla 2 neurônios na camada de saída, responsáveis pela classificação das respectivas classes através das tabelas 1 e 2 .

\begin{tabular}{|c|c|}
\hline CLASSES & COR DA GARRAFA PET \\
\hline A & INCOLOR/TRANSPARENTE \\
\hline B & VERDE \\
\hline C & AZUL \\
\hline D & OUTRA COR \\
\hline
\end{tabular}

Tabela 1, Tabela de classes (Autor)

\begin{tabular}{|c|c|c|c|c|c|}
\hline \multicolumn{3}{|c|}{ ENTRADAS } & \multicolumn{2}{c|}{ SAÍDAS } & \multirow{2}{*}{ CLASSES } \\
\cline { 1 - 5 } X1 & X2 & X3 & Y1 & Y2 & \\
\hline 255 & 255 & 240 & 0 & 0 & A \\
\hline 0 & 255 & 0 & 0 & 1 & B \\
\hline 0 & 0 & 255 & 1 & 0 & C \\
\hline 255 & 255 & 255 & 1 & 1 & D \\
\hline
\end{tabular}

Tabela 2, A dados numéricos (Autor)

\subsection{Dados teóricos}

A sensibilidade relativa dos cones às três cores influenciaram diretamente o desenvolvimento dos dispositivos de exibição coloridos e a criação do espaço de cores RGB como forma de representação das imagens no computador [RUSS, 1992]; [GONZALEZ \& WOODS, 2002].Para descrever, apropriadamente, as cores de uma imagem, o primeiro passo é escolher o espaço de cores a ser utilizado na descrição. Um espaço de cor é uma especificação de um sistema de coordenadas e um subespaço deste sistema onde cada cor é representada por um único ponto (GONZALEZ \& WOODS, 2002). Os principais espaços de cores são o RGB (Red, Green, Blue) e o HSV (Hue, Saturation, Value) (GONZALES \& WOODS, 2002), Sensibilidade relativa dos três tipos de cones presentes na retina, a figura 1.7 mostra essa sensibilidade.

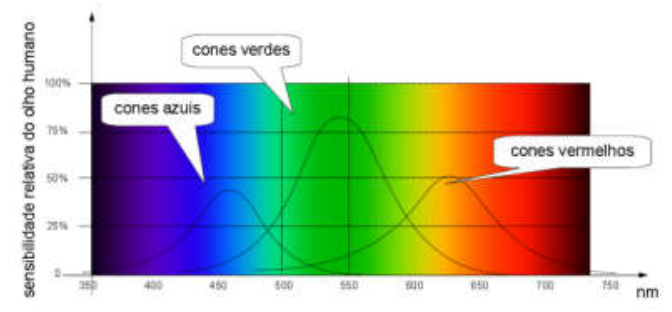

Figura 1.7 Sensibilidade relativa a cores (Autor)

\subsubsection{Espaços RGB}

O espaço de cor RGB é um dos mais aplicados na área de visão computacional, por ser um sistema simples, onde as características físicas do olho humano e dos dispositivos de exibição são mapeadas diretamente [ROGOWITZ ET.AL., 2001]. Este espaço de cor possui três componentes: o primeiro é o Red (vermelho), o segundo é o Blue (azul) e o terceiro é o Green (verde); a

Cor representada consiste na combinação dos três componentes. Uma cor é representada pelos

Valores de suas componentes, normalmente, o intervalo de valores possíveis para cada componente é $[0,255]$, sendo o preto $(0,0,0)$ e o branco $(255,255$, 255). Exemplos da combinação

dos seus componentes podem ser vistos na Figura 2.9, em que é mostrada a combinação das três cores básicas através do diagrama de Venn.

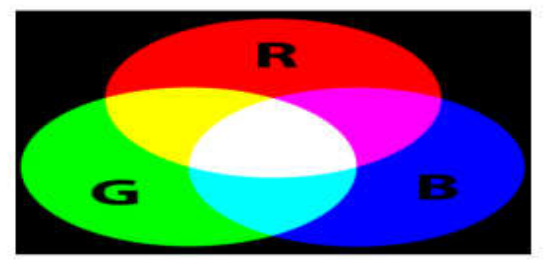

Figura 1.8 Combinações da três cores do espaço RGB ( Autor)

\subsubsection{Espaços RGB}

O espaço de cor HSV também é muito usado em processamento de imagens por ser uma forma mais intuitiva de descrever cores [LONG ET AL., 2003]. O espaço HSV possui três componentes: Hue (mantissa) que representa a cor base, Saturation (luminosidade) que 
representa pura é a cor e Value (brilho) que define o brilho da cor. O intervalo de valores possíveis, a Figura 1.9 mostra o espaço HSV.

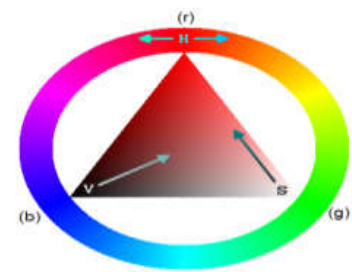

Figura 1.9 Espaço RGB ( Autor)

O nível de diferença pelo observador [MATHIAS \& CONCI, 1998].Ou seja, que sua medida de dissimilaridade seja diretamente relacionada com a dissimilaridade com que ela é percebida pelo observador. Desta forma, o espaço de cor HSV é apropriado para recuperação de imagens, pelo carácter invariante do componente Hue, considerando os fatores de iluminação e de ângulo da câmera [SMEULDERS, WORRING, ET AL., 2000]. Para representar imagens no espaço de cores HSV a partir de imagens representadas no espaço RGB é possível realizar uma transformação matematicamente definida por Gonzalez Cand [WOODS, 2002].

\section{Formulas}
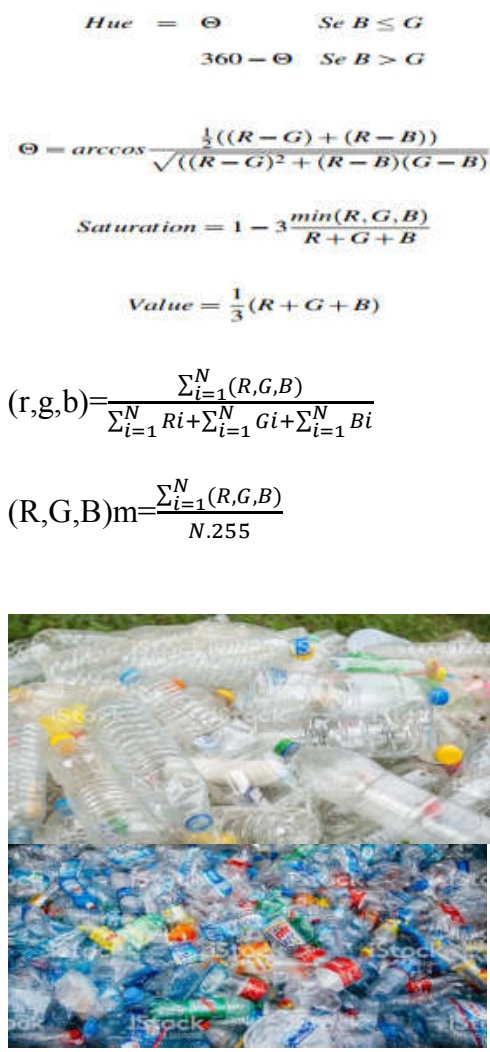

\section{Resultados de implantação}

Foi feito o treinamento da redes coletando as imagens individuais de cada cor de garrafas pet, coletar os dados de cada cores e seus respectivos números da tabela de cores RGB, a partir daí criou um conjunto de amostras para treinar a para ele aprender as variáveis e dificuldades em relação as cores das garrafas, a princípio a redes não teve um rendimento satisfatório, demorando muito para convergir, daí teve que criar pesos afim de diminuir os erros em relação as saídas desejáveis, onde as maiores dificuldades foram em relação a velocidade de processamento a vista que o número de imagens na prática passará muito mais rápido e várias imagens ao mesmo tempo diferente da simulação computacional que as imagens são separadamente, foi feito ajustes em relação taxa de aprendizagem e aumento de neurônios na camada oculta, para tanto ouve um melhor rendimento da rede chegando ao percentual aceitável de acertos de $95 \%$ por cento, pois quando coloca imagens branca perde um pouco de exatidão por isso o $5 \%$ por cento de erro, para tanto na prática esse problema não tem tanta importância pois a garrafa branca não é muito reciclada tem um valor de mercado bem a baixo do valores dessas cores Azul, Verde, transparente, a figura 2.1 mostra o gráfico com a separação de classes.

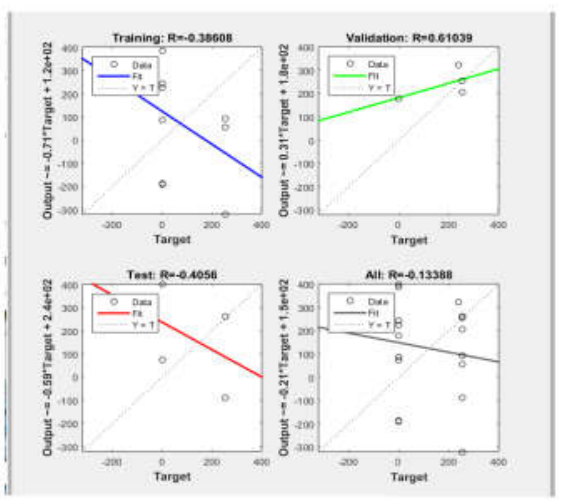

Figura 2.1 Separação de classes ( Autor)

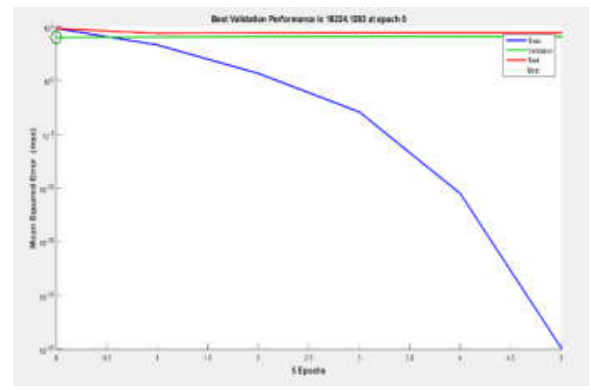

Figura 2.2 Performa da rede ( Autor) 


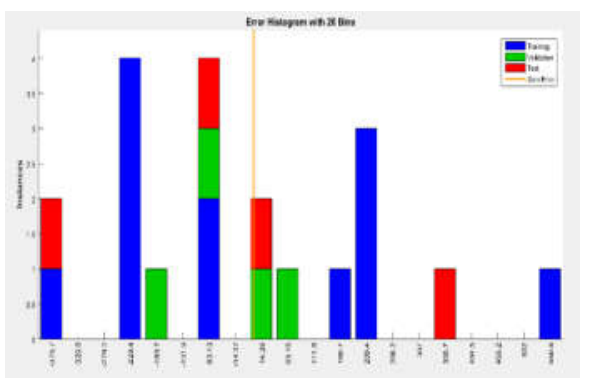

Figura 2.3 Erro da rede ( Autor)

\section{Analise dos resultados}

Os resultados apresentados pela rede foi satisfatório, atendeu as expectativa em treinamento, teste e validação, atingiu os valores e separação de classes desejadas ouve alguns erros quando processa imagens uma atrás das outras as primeiras imagens não sai perfeitamente classificada, mas com os ajustes com seguiu uma boa porcentagem de acertos, 95\%, e 5\% de erro, uma porcentagem bastante grande em relação as possibilidades do sistema, comprovou que o sistema é viável, é lógica que pode ser otimizado para diminuir mais ainda melhorando seu desempenho.

\section{Conclusões}

Para se obter melhores resultados, aumentou-se o grau de complexidade das redes ao introduzir mais neurônios na primeira camada. Porém, se tratando de redes mais simples tal aumento não tornava $\mathrm{o}$ treinamento da rede tão custoso como o ocorrido com a rede individual que avaliava todo o problema, significando em um tempo de treino de em média 20 minutos, apesar de algumas limitações das redes neurais, elas tem várias funções e projetos com uso de redes neurais, para facilitar vários sistemas facilitando em uma porção grandes de aplicações.

\section{Referências bibliográficas}

Abou-Jeyab R. A., Gupta J. R., Gervais J. R., Branchi P. A. e Woo S. S. (2001). Constrained multivariable control of a distillation column using a simplified model predictive control algorithm. Journal of Process Control, vol. 11, pp. 509-517.

J. A. Freeman and D. M. Skapura, 1991, Neural Networks: Algorithms, Applications and Programming ... J. Korbicz, J. M. Koscielny, Z. Kowalczuk and $\mathrm{W}$.

GONZALES, R. C.; WOODS, R. E. Processamento digital de imagens. 3. ed. [S.I.]: Pearson, 2009. Citado 5 vezes nas páginas 3, 4, 5, 6 e 7 .

HAYKIN, S. Redes Neurais. Princípios e Prática. 2. ed. [S.1.]: Prentice Hall, (2001). Citado 5 vezes nas páginas $8,9,10,11$ e 12 .

Alpbaz M., Karacan S., Cabbar Y. e Hapoglu H. (2002). Application of model predictive control and dynamic analysis to a pilot distillation column and experimental verification. Chemical Engineering Journal, vol. 88, pp. 163-174.

Dong J. W., Qian J. X. e Sun Y. X. (2002). Recurrent Neural Networks for Recursive Identification of Nonlinear Dynamic Process. Proc. of the IEEE Int. Conf. Industrial Technology, pp. 794-798.

Hagan M. T. e Menhaj M. B. (1994). Training Feedforward Networks with the Marquardt Algorithm. IEEE Transactions on Neural Networks, vol. 5 , No. 6, pp. 989-993.

GONZALES, R. C.; WOODS, R. E. Processamento

digital de imagens. 3. ed. [S.1.]: Pearson, (2009).

Citado 5 vezes nas páginas 3, 4, 5, 6 e 7 .

HAYKIN, S. Redes Neurais. Princípios e Prática.

2. ed. [S.1.]: Prentice Hall, (2001). Citado

5 vezes nas páginas $8,9,10,11$ e 12 .

SUTTON, R. S.; BARTO, A. G. Reinforcement

Learning: An Introduction. The MIT Press, 1998.

Smeulders , A. W., Worring, M., Santini, S., Gupta, A., \& Jain, R. (2000). ContentBased

Image Retrieval at the End of the Early Years. IEEE Transactions on Pattern

Analysis and Machine Intelligence , 22 (12), 13491380 .

Hen, J.; pappas, T. N.;Mojsilovic, A.; Rogowitz,B. Adaptive image segmentation based on color and texture.In: Internacional conference on imagem processing, (2001).

\section{Anexos}

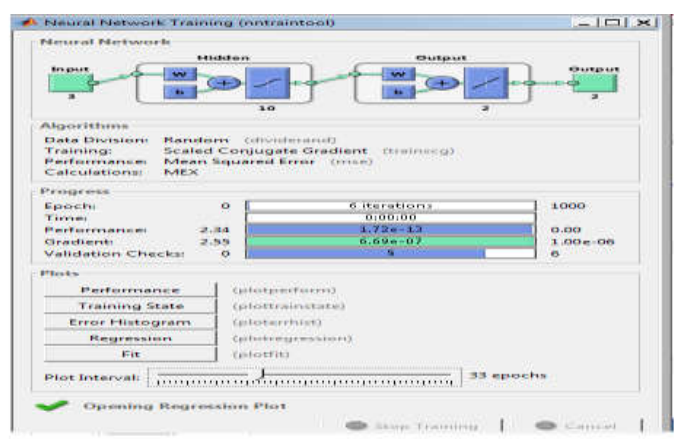



Figura 1. Processo simplificadode destilação de álcool. 

Nota-se, por meio da Figura 7, que o resultado obtido pela segunda abordagem da prova de conceito apresenta alto grau de confiabilidade e precisão para esta solução, bem como ótimo comportamento do sensor, quando submetido a dados adquiridos em diferentes situações do processo de destilação.

Já para o sensor da concentração do álcool hidratado, o EQMapresentado foi de durante a etapa de teste foi de $7.84 \times 10$, visto que foi estabelecido para as etapas de treinamento e validação umEQM de 10 . Assim como nos casos anteriormente comentados, este sensor virtual possui um EQM considerado muito satisfatório para a aplicação em questão. Na Figura 8, ilustra-se uma comparação entre a concentração do álcool, extraída da planta segundo análises laboratoriais, e a concentração medida pelo sensor virtual.
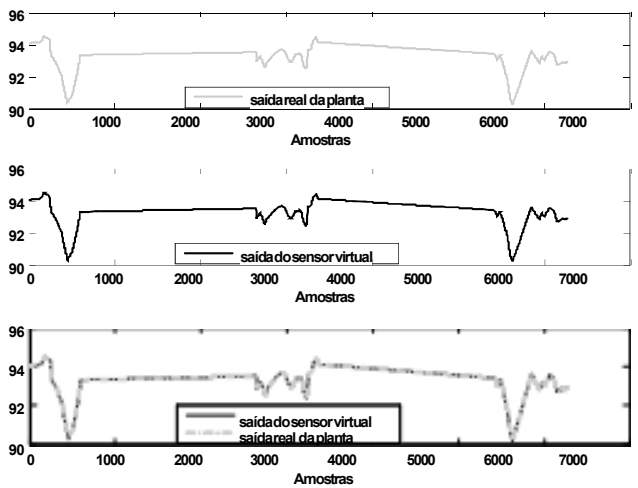

Figura 8. Comparação entre a saída medida pelo sensor virtual e a saída da planta industrial (análises laboratoriais) para a concentração doálcool.

Uma análise dos resultados mostra que as perturbações sofridas pela concentração do álcool são desprezíveis em relação às alterações sofridas pela temperatura da bandeja 18 da coluna A. Esta dinâmica mais lenta favoreceu o desenvolvimento do sensor virtual para a medição deste ponto do processo, uma vez que esta condição não demanda umintervalo de amostragem muito pequeno.

\section{Conclusão}

Conforme os resultados obtidos, pode-se verificar que o sensor virtual apresentou ótimo desempenho, realizando medições instantâneas da planta industrial, de forma precisa e confiável tanto para a temperatura na bandeja 18 da coluna A como para a concentração do álcool. A metodologia proposta possibilitou que este tipo de solução possa ser aplicado em qualquer ponto do processo, sem que haja a necessidade de um sensor físico no local, como foi o caso da concentração do álcool em aparelhos de destilação de etanol. Finalmente, cabese comentar que a técnica apresentada atende a uma importante necessidade industrial, por viabilizar uma solução de baixo custo de implantação e manutenção.

\section{Referências Bibliográficas}

Abou-Jeyab R. A., Gupta J. R., Gervais J. R., Branchi P. A. e Woo S. S. (2001). Constrained multivariable control of a distillation column using a simplified model predictive control algorithm. Journal of Process Control, vol. 11, pp. 509-517.

J. A. Freeman and D. M. Skapura, 1991, Neural

Networks: Algorithms, Applications and

Programming ... J. Korbicz, J. M. Koscielny, Z.

Kowalczuk and W.

GONZALES, R. C.; WOODS, R. E. Processamento

digital de imagens. 3. ed. [S.1.]: Pearson, 2009.

Citado 5 vezes nas páginas $3,4,5,6$ e 7 .

HAYKIN, S. Redes Neurais. Princípios e Prática.

2. ed. [S.1.]: Prentice Hall, 2001. Citado

5 vezes nas páginas $8,9,10,11$ e 12 .

Alpbaz M., Karacan S., Cabbar Y. e Hapoglu H. (2002). Application of model predictive control and dynamic analysis to a pilot distillation column and experimental verification. Chemical Engineering Journal, vol. 88, pp. 163-174.

Dong J. W., Qian J. X. e Sun Y. X. (2002). Recurrent Neural Networks for Recursive Identification of Nonlinear Dynamic Process. Proc. of the IEEE Int. Conf. Industrial Technology, pp. 794-798.

Hagan M. T. e Menhaj M. B. (1994). Training Feedforward Networks with the Marquardt Algorithm. IEEE Transactions on Neural Networks, vol. 5, No. 6, pp. 989-993.

Haykin S. (1999). Neural Networks: A Comprehensive Foundation, Second Edition, Canada.

Jianxu L. e Huihe S. (2002). Soft Sensing Modelling Using Neurofuzzy System Based on Rough Set Theory. Proc. of the American Control Conference, Vol. 1, pp. 543-548.

Policastro C. A., Silva C. B. S., Fernandes R. A. S., Albuquerque A. R. L. e Silva I. N. (2008). Utilização de Sensores Virtuais em Aparelhos de Destilação de Etanol. $12^{\circ}$ Congresso Internacional e Exposição Sul-Americana de Automação, Sistemas e Instrumentação.

Safavi A. A., Nooraii A. e Romagnoli J. A. (1999). A hybrid model formulation for a distillation column and on-line optimisation study. Journal of Process Control, vol. 9, pp. 125-134.

Zanata D. R. P. (2005). Desenvolvimento de Sensor Virtual Empregando Redes Neurais para Medição da Composição em uma Coluna de Destilação. Dissertação de Mestrado, Universidade de São Paulo.

Silva, Ivan N.; Spatti, Danilo H.; Flauzino, Rogério A; Redes Neurais Artificiais para 
engenharia e ciências aplicadas, Artliber, 2010. 